\title{
Rituximab-Rescue Therapy in Autoimmune Neurological Disorders
}

\author{
AK Roy*, Saurabh Sadekar, Rishabh Maloo and Murali S \\ Department of Neurology, Manipal Hospital, Bangalore, India
}

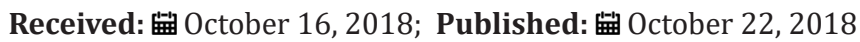

*Corresponding author: Ajit Kumar Roy, Department of Neurology, Manipal hospital, Banglore-560017, India

\section{Opinion}

Demyelinating diseases like Multiple Sclerosis and cases of long segment myelitis, neuromuscular disease like Myasthenia Gravis and Autoimmune Encephalitis have an autoimmune basis and occur in a relapsing remitting course. Achieving and maintaining remission in these patients is quite challenging. Steroids are used for maintaining remission, but its metabolic side effects ultimately are deleterious to the patient. Immunomodulators for remission and Gamma globulin for acute exacerbations are used but are an expensive mode of treatment. All patients may not be able to afford them.

Rituximab is a monoclonal antibody which is used as an immunomodulator in many autoimmune disorders. It selectively depletes B cells having CD 20 on their cell surface. Antigen specific helper T(CD4) cells activate B cells which in turn lead to production of plasma cells generating Ig antibodies found in autoimmune disorders. Hence destruction of the activated B cells plays a vital role in effectiveness of Rituximab. It has three specific modes of action- antibody mediated cytotoxicity, inducing targeted apoptosis and inhibiting B cell dependant granulocyte macrophage colony stimulating factor.

In this study we used Rituximab in patients who could not afford the conventional line of therapy. Two patients of Myasthenia, one patient each of Long segment myelitis, Multiple sclerosis, Autoimmune encephalitis were treated with Rituximab infusions once a week for 4 weeks. The first patient came with unilateral ptosis and diplopia with diurnal variation. He was diagnosed as ocular myasthenia and received Rituximab. There was significant improvement in his symptoms. Second patient had generalized myasthenia and came with a history having underwent thymectomy and being positive for AchR antibody. He was on maintenance therapy with steroids and Azathioprine. But would get symptomatic frequently and had several exacerbations in the past six months. He presented with bulbar symptoms. After receiving 4 cycles of 500 mg of Rituximab there was improvement in neck holding, single breath count, forward arm abduction time and muscle power and the requirement for his previous maintenance therapy decreased significantly.

The long segment myelitis patient had presented with onset of symptoms following an episode of febrile illness. On examination, power was grade $3 / 5$ in all limb muscles, DTRs were brisk, sensory examination showed reduced pain and temperature sensations and loss of joint position sense. After treatment there was improvement in all the affected modalities and he became independent for activities of daily living. Multiple sclerosis patient had developed spasticity in both lower limbs, power of grade 4/5 in upper limbs and grade 2-3/5 in both lower limbs, DTRs were brisk, right planter was extensor. He was wheelchair bound. After treatment with Rituximab there was significant improvement in his disability and Expanded Disability Severity Score. He also became independent for activities of daily living.

Autoimmune encephalitis patient presented low grade intermittent fever for one week during which there was short term memory loss with several episodes of disorientation to time and place. He also developed focal onset seizures which occurred once daily for two days following which it recurred for several times in a day. Following treatment there was no seizure recurrence and memory, cognition also gradually improved. All the patients have received Rituximab and remained in remissions for prolonged periods because of which the need for maintenance steroids reduced significantly or became nil. Between the dosing schedules of Rituximab, the need for maintenance with oral immunosuppressant was eliminated. This shows that Rituximab can be used as a rescue medication in newly diagnosed patients and those who have failed other modalities of therapy and should it also be considered in those patients who cannot afford the standard medical treatment or are suffering from the complications of maintenance therapy. 


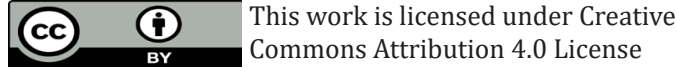

To Submit Your Article Click Here: Submit Article

DOI: $10.32474 / 0 J N B D .2018 .02 .000128$

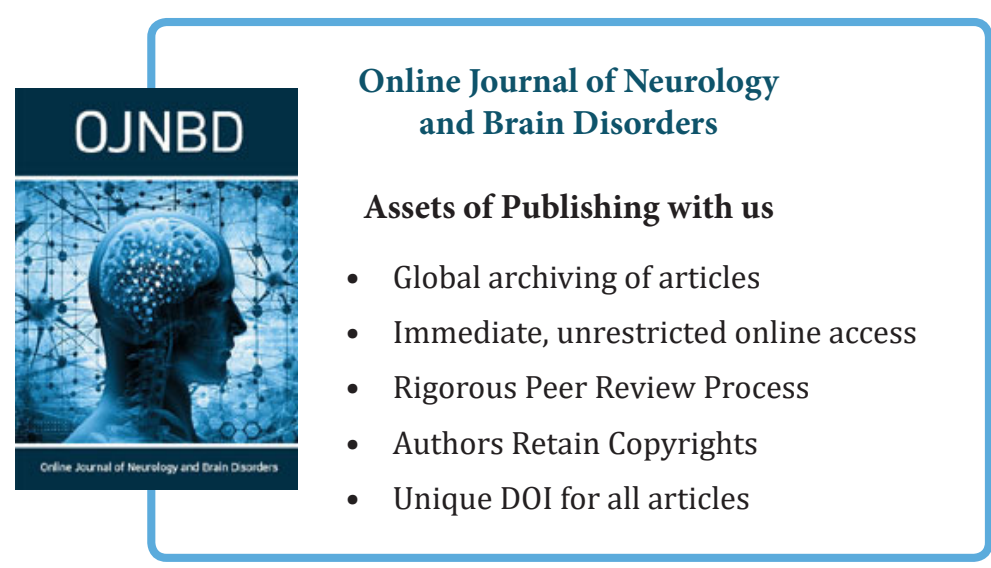

